\title{
An HIV-infected patient with associated, lethal rhinopharyngeal actinomycosis and a rapidly progressing local adenocarcinoma
}

\author{
Roberto Manfredi, , Sergio Sabbatani, Ciro Fulgaro \\ From $16^{\text {th }}$ International Symposium on HIV and Emerging Infectious Diseases \\ Marseille, France. 24-26 March 2010
}

\section{Background}

Solid malignancies, including those with atypical presentations, are increasing 12 years after the introduction of combined antiretroviral therapy (cART), and the differential diagnostic problems may be increased by the eventual concurrence of superinfections.

\section{Methods}

An extremely infrequent episode of associated nasopharyngeal squamous adenocarcinoma plus an underlying actinomycosis occurred in a HIV-infected male patient (p) with a previous diagnosis of AIDS, treated with cART taken with insufficient adherence, so that a satisfactory immune system recovery (as expressed by a CD4+ count persistingly $>400$ cells $/ \mu \mathrm{L}$ ), was in contrast with a lowlevel persistance of detectable HIV viremia, and extensive genotypic drug resistance mutations.

\section{Results}

Interestingly, a number of local and specific risk factors for both neoplastic and infectious disorders were recognized by caregivers (tobacco smoke, long-term inhalatory substance abuse, in particular cocaine, and a half-professional mushroom-truffle search and evaluation also by systematic smelling). Although an appropriate and timely diagnostic workup carried out with repeated, combined computerized tomography, magnetic resonance imaging, and fiberoptic rhinoscopy with multiple biopsy and histopathologic studies, the final diagnosis of a combined, dual neoplasticinfectious pathology occurred only after a demolitive surgical intervention and subsequent pathology studies. Despite a correct antimicrobial therapy, and an associated radiotherapy and cytotoxic chemotherapy schedule, a rapid dissemination of multiple secondary lesions to the brain rapidly led our $\mathrm{p}$ to death.

\section{Discussion}

The particular epidemiological issues, and the imaging and histopathological diagnostic workup of dual illnesses of our HIV-infected p, and its therapeutic and outcome features, are presented and discussed on the ground of the available literature evidences. To the best of our knowledge, no cases of associated actinomycosis plus a local, underlying squamous cell adenocarcinoma of the same ear, nose, and throat district occurred until now in both HIV-infected and also non-HIV-infected p, so that health care professionals should take into careful consideration even a dual etiology, when facing $\mathrm{p}$ with rhinopharyngeal mass lesions, with multiple risk factors for different diseases.

Published: 11 May 2010

* Correspondence: Roberto.manfredi@unibo.it

Infectious Diseases, S. Orsola Hospital, Bologna, Italy

i:10.1186/1742-4690-7-S1-P40

Cite this article as: Manfredi et al:: An HIV-infected patient with associated, lethal rhinopharyngeal actinomycosis and a rapidly progressing local adenocarcinoma. Retrovirology 2010 7(Suppl 1):P40. 